\title{
SU DUE IMPROBABILI CITAZIONI DAI KORINTHIAKÁ DI EUMELO $(F$ 2; 8 BERNABÉ $=12$ DAVIES): UN NUOVO FRAMMENTO LIRICO DI AUTORE INCERTO (ESCHILO?) E UN ORACOLO SIBILLINO
}

\author{
EUGENIO AMATO
}

La atenta consideración del discurso corintio de Favorino (ps.-Dio Chrys., Or. 37) ha permitido llegar a una propuesta de lectura nueva que aclara el sentido de dos fragmentos anónimos citados en Cor. 11-13, corrientemente atribuídos a los Corinthiaca de Eumelo. Por otra parte, el análisis exegético, linguístico y métrico de esas citas sugiere la posibilidad de atribuirlas a la poesía lírica griega, tal vez a las Isthmiastai de Esquilo, y a los oráculos sibilinos.

Palabras clave: Esquilo; dicurso Corintio; pentámetro dactílico; poesía épica; Eumelo; Favorino; griego; métrica griega; poesía lírica griega; Pausanias; Dión Crisóstomo; oráculo sibilino.
The attentive consideration of Favorinus' Corinthian speech (Ps.-Dio Chrys., or. XXXVII) has permitted to obtain a new reading proposal that clears the meaning of two anonymous fragments quoted in Cor., 11-13, fluently assigned to Eumelus' Korinthiaká. Further more, the exegetic, linguistic and metric analysis of the quotations suggest the attribution to Greek lyric poetry, maybe the Aeschylus' Isthmiastai, and to Sybilline oracles.

Keywords: Aeschylus; Corinthian speech; Dactylic pentameter. Epic poetry. Eumelus. Favorinus. Greek. Greek metric. Greek lyric poetry. Pausanias. Dio Chrysostom. Sybilline oracle.

Entro subito in medias res: nella Corinthiaca da Favorino (Ps.-Dio Chrys., Or. XXXVII 11-13 ${ }^{1}$ ) sono inserite, a breve distanza, due citazioni

1 L'edizione attualmente di riferimento è quella curata da A. Barigazzi, Favorino di Arelate. Opere, Introduzione, testo critico e commento, Firenze, 1966 (d'ora in poi Barigazzi). L'attribuzione a Favorino della Corinthiaca risale al Niebuhr (Römische Geschichte, I, Berlin, 1811, p. 118) e all'Emperius (De oratione Corinthiaca falso Dioni Chrysostomo adscripta, Brunsvigae, 1832 = Opuscula philologica et historica, ed. F.G. Schneidewin, Gottingae, 1847, pp. 18-49). Dopo l'edizione del Barigazzi, nessuno ha più contrastato tale paternità (per la letteratura precedente, vedi Barigazzi, p. 298); eppure, per

EMERITA. Revista de Lingüística y Filología Clásica (EM) — LXX 1, $2002 \quad$ pp. 45-68 
poetiche di autore ignoto, che il Barigazzi per primo, nella sua edizione favoriniana del $1966^{2}$, ha attribuito, in via definitiva, ai Korinthiaká di

come talora sono citati i passi di quest'orazione nei diversi contesti, si ha l'impressione che a nulla sia valsa tanta letteratura: vedi, ad esempio, già prima dell'edizione del Barigazzi, H.W. Parke, A History of the Delphic Oracle, Oxford, 1939, p. 136, n. 1; E. Lobel-D. Page, Poetarum Lesbiorum fragmenta, Oxford, 1955, app. di p. 96; F. Täger, Charisma. Studien zur Geschichte des antiken Herrscherkultes, I, Stuttgart, 1957, p. 175, n. 18; P. Waltz, Anthologie Grecque, IV, Paris, 1960, app. di p. 119 (epigr. 153), 185 (epigr. 304) e 202 (epigr. 347); e, in seguito, M. Gigante, Diogene Laerzio. Vite dei filosofi, II, Roma-Bari, $1998^{3}$, p. 520, n. 155; E.-M. Voigt, Sappho et Alcaeus, Amsterdam, 1971, app. di p. 139; J.R. Ellis, Philipp II and Macedonian Imperialism, London, 1976, p. 296, n. 95; G.T. Griffith in A History of Macedonia, ed. by N.G.L. Hammond - G.T. G., II, Oxford, 1979, p. 611, n. 4; F. Fuhrmann, Plutarque. Oeuvres morales, t. IX-2 ${ }^{2}$ Paris, 1978, p. 165; G. Lachenaud, Plutarque. Oeuvres morales, t. XII- ${ }^{1}$, ibid., 1981, app. di p. 180; D.A. Campbell, Greek Lyric, I, Cambridge, Mass. - London, 1982, p. 159; M. Capone Ciollaro, Dione Crisostomo. Diogene o Discorso Istmico (or. 9), Napoli, 1987, p. 41; D. Musti - M. Torelli, Pausania. Guida della Grecia. Libro II. La Corinzia e l'Argolide, Milano-Verona, 1986, p. 207; D. Asheri, Erodoto. Le Storie (libro I). La Lidia e la Persia, ibid., $1991^{3}$ (1988), app. di p. 27; F. Lasserre, Sappho. Une autre lecture, Padova, 1989, p. 78; S.M. Medaglia, Erodoto. Le Storie (libro III). La Persia, Milano - Verona, 1990, app. di pp. 31-32; l'addendum di S. Lilla a E. Fraenkel, Orazio, trad. it. Roma, 1993, p. 521; A. Caiazza, Plutarco. Precetti politici, Napoli, 1993, app. di p. 167; L. Canfora - A. Corcella, «La letteratura politica e la storiografia», in AA.VV., Lo spazio letterario della Grecia antica, I. 1, Roma, 1994, p. 439; G. Nenci, Erodoto. Le Storie (libro V). La rivolta della Ionia, Milano - Verona, 1994, app. di p. 107.

2 Tutte le opere di Favorino sono ora in corso di riedizione a cura di chi scrive, con la collaborazione di Adele Tepedino Guerra per il de exilio, nella "Collection des Universités de France": vedi gli studi preparatori di A. Tepedino Guerra, «Un nuovo frammento pindarico dell'Inno ad Ammone? Pap. Vat. Gr. 11, col. VII 44-46 (Favorino, Sull'esilio)», REG 110, 1997, pp. 353-361; Ead., «Nuove letture del Pap. Vat. Gr. 11 (Favorino, Sull'esilio)», ZPE 131, 2000, pp. 29-39; Ead., «Per una nuova edizione del De exilio di Favorino (Pap. Vat. Gr. 11)», in Papyrologica Florentina, Atti del XXII Convegno Internazionale di Papirologia (Firenze 23-29 agosto 1998), Firenze, 2001 (in bozze), e di E. Amato, Studi su Favorino. Le orazioni pseudo-crisostomiche, Salerno, 1995; Id., Per la ricostruzione del $\pi \varepsilon p \grave{~ \gamma \eta ́ p \omega s ~ d i ~}$ Favorino di Arelate, Salerno, 1999; Id., Alle origini del «corpus Dioneum»: per un riesame della tradizione manoscritta di Dione di Prusa attraverso le orazioni di Favorino, Salerno, 1999; Id., «De Favorini Arelatensis orationis Corinthiacae codicibus», Latinitas 34, 1, 1996, pp. 7-31; Id., «Appunti per la fortuna di Favorino a Bisanzio (con un'appendice sulla Pro balneis)», REG 112, 1, 1999, pp. 259-269; Id., «Pour Diogène Laërce VIII, 83 (Favorinus, fr. 74 Barigazzi)», EMC 43, n.s. 18, 2, 1999, pp. 397-400; Id., «Cristoph M. Wieland lettore di

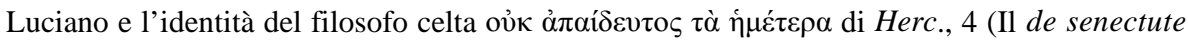
di Favorino e l'esilio a Chio)», in Weimar, le Letterature Classiche e l'Europa del 2000, Atti delle giornate di studio (Liceo Classico "F. De Sanctis" di Salerno: 27 settembre 1999-31 gennaio 2000), a cura di E. A., A. Capo, D. Viscido, Salerno, 2000, pp. 87-125; Id., 
Eumelo $^{3}$, l'antichissimo cantore della preistoria mitica di Corinto $^{4}$, il quale, come a giusto titolo è stato scritto, «esercitò una sorta di monopolio poetico simile a quello di Omero per la Ionia» ${ }^{5}$.

«Favorino in Diogene Laerzio: la nuova edizione teubneriana di M. Marcovich», ibid., pp. 309-320 (appare ora con alcuni tagli in Gnomon); Id., «Adversaria critica in Favorinum Arelatensem», Latinitas 48, 4, 2000, pp. 275-287; Id., «Favorino, Sul 'proprio' esilio», ZPE

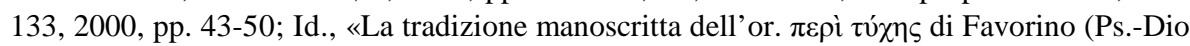
Chrys., or. 64)», RHT 30, 2000, pp. 93-108; Id., «Favorino e la critica scettica alla divinazione artificiale», Pomoerium 4, 2000; Id., «Favorino nell'Anthologia Palatina (e un epigramma contestato a Meleagro)», Scholia 10, 2001 (in stampa); Id., «Sentenze di Favorino in tre manoscritti inesplorati di Oxford, Cambridge e Londra (con una nota al fr. III Callanan Bertini Malgarini)», di prossima pubblicazione in $R h M$; «Le 'tournées de conférences' di Favorino: nuove ipotesi sulla città del De fortuna», che apparirà in Athenaeum 91, 2003. È rimasto finora tale il progetto di una nuova riproduzione del de exilio, annunciato e discusso anni fa da R. Pintaudi, «"Primum legere...": a proposito di un progetto di riproduzione in facsimile del Pap. Vat. Gr. 11», APapyrol 1990, 2, pp. 21-25.

3 Mentre i quattro versi della Sibilla, di cui mi accingo a discutere, sono considerati tali fin dall'edizione dionea del Morel (Dionis Chrysostomi orationes LXXX..., Lutetiae, 1604, p. 458 ), a ritenere anche il verso isolato relativo a Briareo una citazione poetica fu per primo l'Emperius (Dionis Chrysostomi Opera Graece..., II, Brunsvigae, 1844, p. 523), il quale, pur senza indicare il presunto autore ovvero le ragioni di tale scelta, è stato seguito da tutti gli editori successivi. L'Arnim, nell'app. ad l. della sua edizione (Dionis Prusaensis quem vocant Chrysostomum quae extant omnia, II, Berolini, 1986, p. 19), annota «versus unde sumptus sit nescio»; di qui H.L. Crosby, Dio Chrysostom with an English Translation, IV, Cambridge, Mass. - London, 1946, p. 13, n. 3: «Author unknown» e G. del Cerro Calderón, Dión de Prusa. Discursos XXXVI-LX, Madrid, 1997, p. 50, n. 22: «La cita es de autor desconocido».

4 Per la cronologia di Eumelo (metà VIII sec. a.C.), vedi in particolare C.M. Bowra, «Two Lines of Eumelus», $C Q$ n.s. 13, 1963, pp. 145-153 (= On Greek Margins, Oxford, 1970, pp. 46-58). I frammenti dei Corinthiaca sono attualmente disponibili nell'edizione teubneriana di A. Bernabé (Poetarum epicorum Graecorum testimonia et fragmenta, I, cum appendice iconographica a R. Olmos confecta, Stutgardiae-Lipsiae, 1996 ${ }^{2}$ 1987¹, pp. 108112), che ha sostituito la vecchia edizione del Kinkel (Epicorum Graecorum fragmenta, I, Lipsiae, 1877), e in quella di M. Davies (Epicorum Graecorum fragmenta, Göttingen, 1988, pp. 96-101; andrà segnalato che per errore il Davies a p. 101 indica l'orazione di Favorino come «[Dio Chrysostom.] or. 20»). Edizioni parziali dei soli Korinthiaká si erano avute in passato ad opera di E.G. Wilisch (Über die Fragmente des Epikers Eumelus, Nachrichten über das Johanneum in Zittau, 1875) e di F. Jacoby (Die Fragmente der griechischen Historiker, III B, Leiden, 1950, pp. 378-381, nr. 451). Sempre al Bernabé si deve una traduzione spagnola per la benemerita "Biblioteca Clásica Gredos" (Fragmentos de épica griega arcaica, Madrid, 1979: pp. 250-267 per Eumelo).

5 Fondamentali, in tal senso, lo studio di G.L. Huxley, Greek Epic Poetry from Eumelos to Panyassis, London, 1969, praes. pp. 61-79 e quello di J.P. Salmon, Wealthy Corinth, 
Invero, le motivazioni di tale scelta si leggono in maniera diffusa e dettagliata in un contributo dello stesso Barigazzi, apparso contemporaneamente su RFIC 94, 2, 1966, pp. 129-148 («Nuovi frammenti dei Corinthiaca di Eumelo»), cui l'editore rimanda nel commento della sua edizione 6 .

La proposta, almeno per quanto mi consta, è stata finora pacificamente accolta dagli studiosi ${ }^{7}$ ed il locus favoriniano viene inserito tra i frammenti

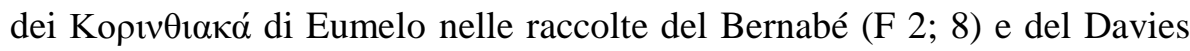
(F 12). Mentre, però, il primo studioso inserisce le due citazioni favoriniane tra i luoghi certi del poema epico del cantore di Corinto, il Davies preferisce, più cautamente, stamparle tutte e due assieme tra i fragmenta

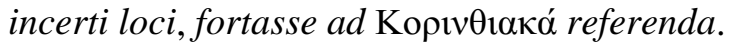

Personalmente (anticipo così le mie conclusioni), ritengo che, se certo non si può del tutto escludere che Eumelo sia tra le fonti di Favorino ${ }^{8}$, è del tutto improbabile che le due citazioni poetiche derivino dai Korinthiaká di quel poeta.

Oxford, 1984, pp. 38-41; cf., inoltre, E. Bethe in RE VI, 1, 1907, s.v. «Eumelos» (nr. 12), coll. 1080-1081; M. Untersteiner, «Eumelo di Corinto», Antiquitas 6-7, 1951-1952, pp. 3-13 (= Scritti minori. Studi di letteratura e filosofia greca, Brescia, 1971, pp. 165-179); F. Lasserre, «L'historiographie grecque à l'époque archaïque», QS 4, 1976, pp. 113-142; J. Latacz, «Eumelos, frühgriechischer Literat aus Korinth», in Der Neue Pauly. Enzyklopädie der Antike, hsg. von H. Cancik und H. Schneider, IV, Stuttgart-Weimar, 1998, pp. 249-250; Ch. Harrauer, «Der korinthische Kindermord - Eumelos und die Folgen», WS 112, 1999, pp. 5-28. L'espressione tra virgolette è di F. De Martino in F. D.M. - O. Vox, Lirica greca, I. Prontuari e lirica dorica, Bari, 1996, p. 112.

6 Barigazzi, pp. 322-323.

7 Cf., e.g., G. D’Ippolito, «Epici greci minori», in Dizionario degli Scrittori Greci e Latini, diretto da F. Della Corte, vol. I. A-Epic, Milano, 1990, p. 724; P. De Fidio, «Un modello di mythistoríe: Asopia ed Efirea nei Korinthiaká di Eumelo», in F. Prontera (a cura di), Geografia storica della Grecia antica: tradizioni e problemi, Roma, 1991, pp. 233-263. G. del Cerro Calderón, nella già menzionata traduzione spagnola, stranamente continua a riproporre l'anonimato, dimenticando del tutto il contributo del Barigazzi.

${ }^{8}$ Per un ritratto dell'autore, oltre l'importante introduzione del Barigazzi (una sintesi apparsa postuma si legge in ANRW II, 34.1, 1993, pp. 556-581), vedi di recente L. HolfordStrevens, «Favorinus: the man of paradoxes», in J. Barnes - M. Griffin (edd.), Philosophia Togata II. Plato and Aristotle at Rome, Oxford, 1997, pp. 188-217 (da tenere presente dello stesso anche l'Aulus Gellius, Chapel Hill, 1989 [London, 1988], pp. 72-103) e S. Follet, «Favorinus d'Arles», in R. Goulet (ed.), Dictionnaire des Philosophes Antiques, III, Paris, 2000, pp. 418-422. 
Sarà utile, a questo punto, trascrivere l'intero contesto citante (che rappresenta, in pratica, il frammento com'è edito nel menzionato corpus epico del Davies), in modo da rendere chiare le diverse difficoltà esegetiche. Avverto che, per praticità, riproduco il testo e l'apparato del Barigazzi ${ }^{9}$, senza entrare nel merito delle numerose questioni critico-testuali inerenti al passo, quale, invece, si potrà leggere nella mia prossima edizione per la C.U.F. ${ }^{10}$.

\section{Favorin., Corinth., 9-16 (p. 304, 22-305, 30 Barigazzi)}

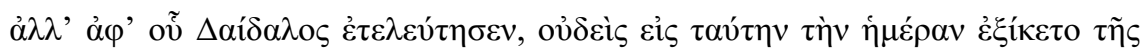

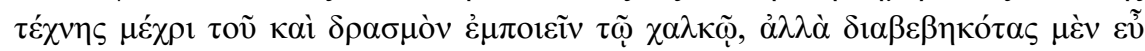

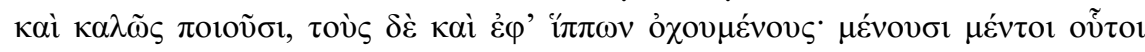

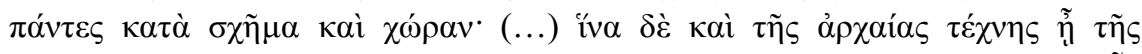

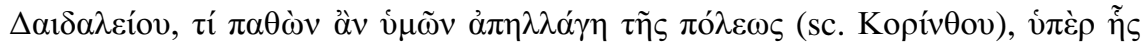

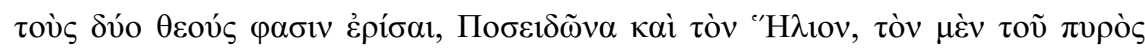

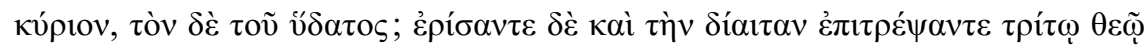
$\pi \rho \varepsilon \sigma \beta v \tau \varepsilon \dot{\varepsilon} \rho \varphi$, ỡ

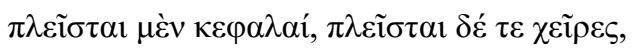

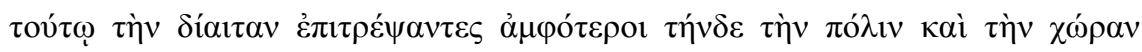

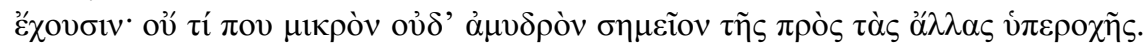

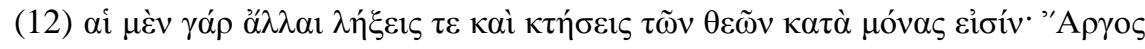

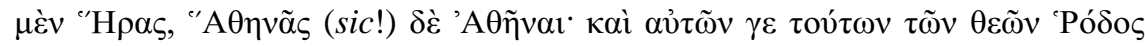

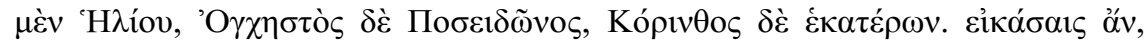

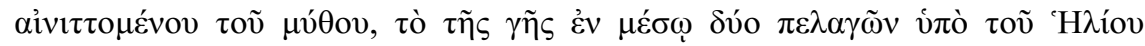

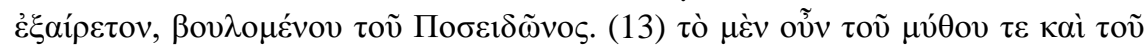

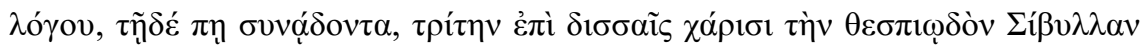

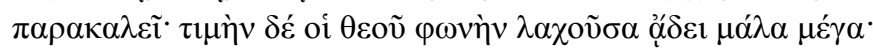

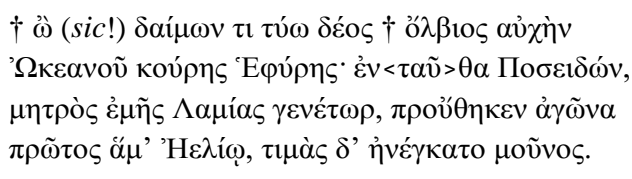

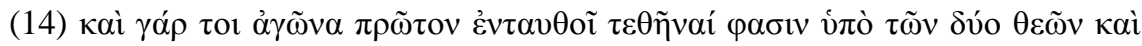

9 Naturalmente nell' apparato indico solo le varianti ovvero le correzioni degli studiosi ai versi in questione. Per un refuso, credo, nella raccolta del Davies è segnato il punto fermo

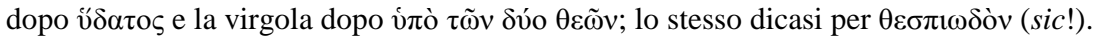

${ }^{10}$ Ho anticipato alcune note nei miei Studi su Favorino, op. cit., pp. 43-80 (in part. pp. 53-56) e negli «Adversaria critica in Favorinum Arelatensem», cit., in part. pp. 277-278; vedi, inoltre, Alle origini del «corpus Dioneum», op. cit., pp. 67-75. 


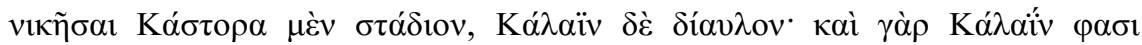

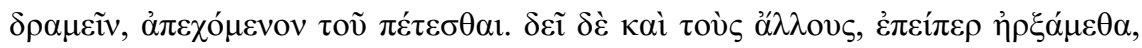

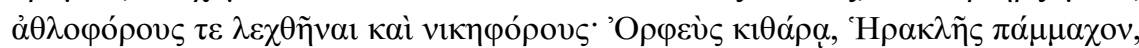

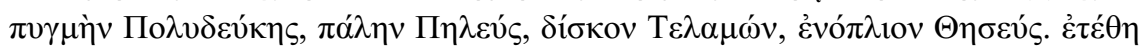

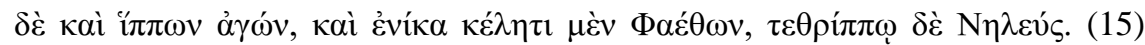

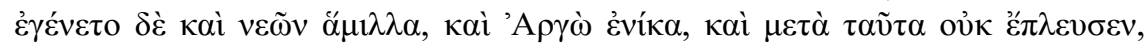

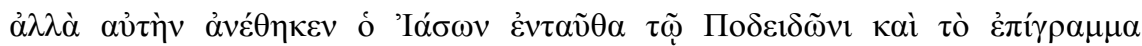

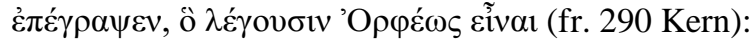

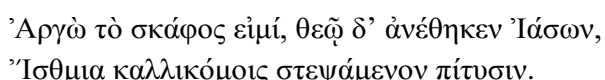

(16) ö

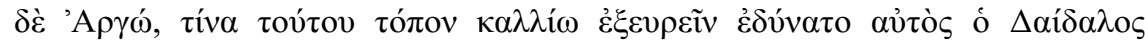

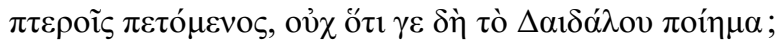

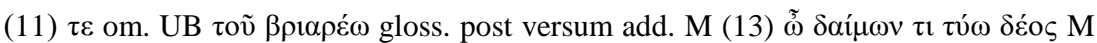

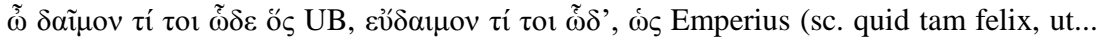

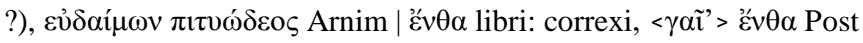

Ad eccezione dell'ultimo distico, che ha chiaramente una fonte diversa, gli altri cinque versi, secondo il Barigazzi, riguardano le stessa leggenda (la contesa di Posidone ed Elio per il possesso di Corinto e l'istituzione dei giochi istmici) e proverrebbero, quindi, dal medesimo poema di un poeta arcaico che di quella città mise in versi le mitiche e lontane origini ${ }^{11}$.

Il confronto con le testimonianze di Pausania (in particolar modo i passi di II 1.1; 1.6 e II 4.6), dove, richiamando l'autorità di Eumelo, è narrata la stessa versione della gara delle due divinità, di cui, come in Favorino, è fatto giudice Briareo (le parole $\pi \lambda \varepsilon \tilde{\sigma} \sigma \tau \alpha l-\chi \varepsilon i \tilde{\rho} \varepsilon \varsigma$, come chiarisce anche una glossa in $\mathrm{M}^{12}$, si riferiscono senza dubbio al centimano figlio di Urano e Gea), ed

11 La conclusione del Barigazzi è abbracciata totalmente dal Bernabé, il quale così annota: «Eumelo tribuit Barigazzi ... credens, mea sententia recte, Eumelum Favorini fontem esse et in ceteris narratis de Neptuni et Solis lite et de Briarei arbitrio» (Poetarum epicorum Graecorum, cit., p. 108, ad l.).

${ }^{12}$ Si tratta del Leidensis B.P. Gr. 2c (un tempo Meermannianus Lugdunensis 67), della prima metà del XVI secolo. La glossa compare anche nel Laurentianus plut. 81, 2 (siglato E) e viene stampata, a torto, nell'editio princeps del Torresano (Dionis Chrysostomi Orationes LXXX..., Venetiis, s.d. [1551?]) e in quelle successive del Morel, del Reiske (Dionis

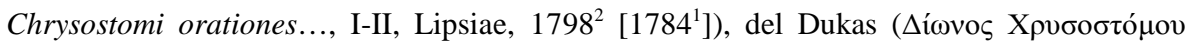


Efira, la prima che abitò Corinto e diede il nome più antico alla città, è detta figlia di Oceano, lasciano concludere allo studioso che «i versi citati da Favorino sono di Eumelo» (p. 134).

Orbene, prima di affrontare il problema delle fonti di Favorino e Pausania e delle possibili interazioni tra i due testi, sarà necessario, credo, soffermarsi sul metro delle due citazioni, che, stranamente ed erroneamente, il Barigazzi (p. 132) interpreta come esametri epici regolari.

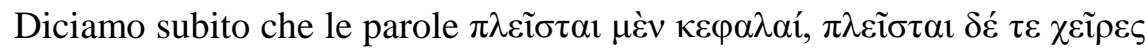
non rappresentano affatto un esametro: manca un intero piede!

Piuttosto, la costruzione alternata $\mathrm{ABA}^{\prime} \mathrm{B}^{\prime 13}$, di prima lettura, potrebbe dare l'impressione di un elegiaco, se non fosse per il fatto che il secondo hemiepes presenterebbe la contrazione dei bicipitia (si dovrebbe in ogni caso ipotizzare qualche 'allungamento metrico' ${ }^{14}$ o correggere, ad es., $\tau \varepsilon$ della tradizione in $\tau o$ ), fenomeno rarissimo e, laddove attestato (per lo più in epigrafi), da attribuire all'opera di versificatori maldestri ${ }^{15}$. Del resto, l'uso stichico di questo tipo di "pentametro", com'è noto, è attestato solo in alcune iscrizioni ${ }^{16} \mathrm{e}$ in un epigramma di Filippo di Tessalonica ${ }^{17}$.

Che si tratti, allora, di un pentametro dattilico catalettico in disyllabum con contrazione spondaica nel primo e nel terzo piede?

L'uso lirico del dattilo è variamente attestato nella poesia arcaica e tardo arcaica e nel dramma attico ${ }^{18}$. Diversamente da Omero (e dall'epica in gene

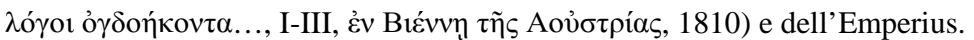

13 Cf. D. Korzeniewski, Griechische Metrik, Darmstadt, 1968, pp. 38-39.

14 Vedi B. Snell, Metrica Greca, trad. it. a cura di Fr. Bornmann, Firenze, 1990² (Göttingen $1962^{3}$ ), p. 78.

15 Vedi G.B. D’Alessio, «Sull'epigramma dal polyandrion di Ambracia», ZPE 106, 1995, p. 23 e n. 4.

16 Cf. W. Peek, Griechische Vers-Inschriften, I, Berlin, 1955, 1400, 1805; M.C. Martinelli, Gli strumenti del poeta. Elementi di metrica greca, Bologna, 1995, p. 293.

17 A.P. XIII 1; cf., inoltre, A.P. IX 485 (= Hld. III 2.4), su cui vedi B. Palumbo Stracca, «Lettura critica di epigrammi greci (I)», BollClass 6, 1985, p. 72.

${ }_{18}$ Per i dattili lirici resta fondamentale lo studio di E. Fraenkel, «Lyrische Daktylen», RhM 72, 1917, pp. 161-197 (ora in Kleine Beiträge zur Klassischen Philologie, I, Roma, 1964, pp. 165-196). Cf., inoltre, Snell, op. cit., p. 26-33; A.M. Dale, Collected Papers, 
re), la dissoluzione dello schema dattilico formato dal $\mu \varepsilon ́ \tau \rho o v /-\smile \backsim-\backsim \sim /$ e, quindi, la possibilità dell'impiego del solo piede / - ৩/ come unità semplice, accostabile anche in numero dispari, compare con una certa evidenza in Stesicoro ${ }^{19}$ e in Ibico $^{20}$, contrariamente ad Alcmane, il quale sembra che nella sua opera utilizzasse solo numeri pari di dattili (tetrametri, esametri, ottametri): «questo fa supporre che, non diversamente dall'esametro epico, anche in questi dattili cantati fossero sentiti come un'unità volta per volta due dattili» ${ }^{21}$. Eppure, come ha ricordato C.O. Pavese ${ }^{22}$, versi costituiti da un pentametro si trovano, nella citarodia, anche in Terpandro $(P M G 698.2=\mathrm{fr}$. 3 Gostoli) e proprio nella Gerioneide di Stesicoro $(S 11,16.25 ; S 14.4$, ecc.) ed in Ibico (6.65), nella lirica corale, in Pindaro $(P .3 \text {, str. } 4)^{23}$ e, guarda caso, in Eumelo, il quale, nel prosodio all'Apollo di Delo $(P M G 696,1)$, ne offre l'esempio più antico. Quanto alla tragedia, l'unico a farne uso sembra essere Eschilo (Ag. 105-106; 124; 139) ${ }^{24}$ e, dalla parodia che ne fa Aristofane in Ran. $816^{25}$, si presume che questo verso fosse tipico del nomos citaro-

Cambridge, 1969, p. 185-209. Anche se si preferisce la soluzione con dattili trisillabici, non mancano gli esempi di sostituzione dei brevia doppi con sillabe lunghe. Stesicoro, tuttavia, per evitare una sequenza con più di tre sillabe lunghe, che inciderebbe sul ritmo, sembra non tollerare la presenza di sostituzioni spondaiche in piedi adiacenti (cf. P. Lerza, Stesicoro. Tre studi. Frammenti con traduzione a fronte, Genova, 1982, pp. 22-23). Il caso limite è rappresentato da due versi olospondaici attribuiti a Terpandro (cit. infra).

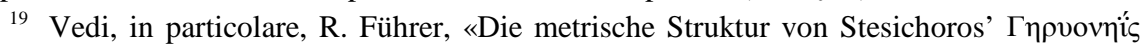
(P. Ox. 2617)», Hermes 96, 1968, pp. 675-684; M.W. Haslam, «Stesichorean Metre», QUCC 17, 1974, pp. 11-24. Sul problema dell'innovazione (se di innovazione si tratta) apportata in tal senso da Stesicoro, vedi la sintesi di Lerza, op. cit., p. 22 (p. 103, n. 7 per la bibliografia precedente); da tenere presenti, comunque, le conclusioni di B. Gentili, Poesia e pubblico nella Grecia antica, Bari, $1989^{2}$, pp. 164-165, 239. Invero, che Stesicoro non abbia composto poesia esametrica, pur avendo come modello l'epica arcaica e trattando nei suoi componimenti materia eroica, è detto esplicitamente da Dione Crisostomo (or. 55, 6) nel noto accostamento con la poesia di Archiloco.

20 Da Ibico prende il nome il metrum ibycium, che Servio spiega ora come eptametro acataletto ora come eptametro ipercataletto: vedi De Martino, op. cit., p. 294.

${ }^{21}$ Così Snell, op. cit., p. 26.

22 «Il più antico frammento di lirica corale greca», in AA.VV., Filologia e forme letterarie. Studi offerti a Francesco Della Corte, Urbino, 1987, I, pp. 53-57 (54).

${ }^{23}$ Per una possibile interpretazione di Pi., I. 1, ep. 1 come $\pi \varepsilon v \tau \alpha ́ \mu \varepsilon \tau \rho o v ~ \delta \alpha \kappa \tau v \lambda \imath \kappa o ́ v$

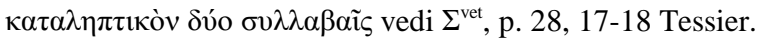

${ }^{24}$ Un esempio di pentametro catalettico in syllabam offre Eur., Hel. 384.

25 Sull'argomento, vedi C. Prato, I canti di Aristofane, Roma, 1962, p. 307. 
$\operatorname{dico}^{26}$ (come del resto lo stesso Aristofane chiarisce in Ran. 1128-1129, definendo citarodico il canto eschileo) ${ }^{27}$.

Detto questo, risulta manifesto, già dal solo (ed ineludibile) punto di vista metrico, l'impossibilità di attribuire la citazione ai Korinthiaká di Eumelo, redatti in forma squisitamente esametrica, come si deduce, del resto, dagli otto versi sopravvissuti in $\Sigma$ Pi., O. XIII 74 (F 3 Bernabé $=2^{\mathrm{A}}$ Davies $^{28}$ ).

$* * *$

Lasciamo in sospeso, per il momento, la questione dell'eventuale attribuzione del nuovo pentametro lirico e passiamo, così, agli altri quattro esametri, quelli che, come scrive Favorino, con voce oracolare pronunciava la Sibilla, cioè Libyssa, la figlia di Zeus e Lamia, nipote di Posidone per parte della madre, la più antica Sibilla a sentire Plutarco (de Pyth. or. 398C), che vaticinava dall'alto di una rupe presso Delfi ${ }^{29}$.

Il Casaubon, nella Diatriba pubblicata in appendice all'edizione morelliana, proponeva, senza veri argomenti, di attribuire il breve componimento a Pindaro: «Locus de Sibylla ex aliquo poëta lirico depromptus est: ni fallor, ex Pindaro, qui oraculi mentionem fecerat, quod statim Dio subiicit ${ }^{30}$.

${ }^{26}$ Cf. B. Gentili, in B.G. - P. Giannini, «Preistoria e formazione dell'esametro», QUCC 26, 1977, p. 36 .

27 In età ellenistica, l'uso del pentametro dattilico catalettico in disyllabum ritorna in Simia di Rodi (fr. 17 Powell), tanto che il verso dal nome poeta prende il nome di «simieo»: cf. Martinelli, op. cit., p. 181.

28 Sul passo vedi ora O. Poltera, «Simonide, Eumélos et la [sic!] «Korinthiaka» (Simon. 545 PMG): un fragment irrécupérable?», EMERITA 65, 1997, pp. 311-319.

${ }^{29}$ Cf., inoltre, SVF II, p. 348, fr. 1216; Varr. ap. Lact., Div. Inst., I 6.7-8 = Heracl. Pont., fr. 131a Wehrli; F. Lübker, Lessico ragionato della Antichità Classica, trad. it. a cura di C.A. Murero, Roma, 1898 (Leipzig $1882^{6}$ ), rist. anast. Bologna, 1989, s.v. «Sibilla», p. 1118; Rzach in RE II $\mathrm{A}_{2}$, s.v. «Sibyllen», coll. 2089-2090; M. Delcourt, L'oracle de Delphes, Paris, 1955, pp. 220-222. Pausania (X 12.1) forse per errore chiama questa stessa Sibilla Erofile, confondendola con l'omonima Erofile di Eritrea, che ebbe il soprannome di Sibilla dopo esser divenuta profetessa e della quale, incostante nei suoi spostamenti come le altre sibille, si danno come patria diverse città, tanto da essere identificata contemporaneamente con la Sibilla di Sardi, Troia, Samo, Delfi, Cuma (cf. Plut., ibid. 401B; de sera num. vind. 29, 566D; sch. ad Plat., Phaedr. 244B; U. von Wilamowitz-Möllendorff, Der Glaube der Hellenen, II, Berlin, 1932, p. 34, n. 1). Nella medesima confusione cade il Barigazzi (Barigazzi, p. 322) e il Bernabé (Poetarum epicorum Graecorum, cit., p. 111, ad l.).

30 I. Casaubonus, In Dionem Chrysostomum diatriba av̉ 
L'osservazione, pur nella sua artificiosità ${ }^{31}$, è sfuggita al Barigazzi, così come quella avanzata da H.W. Parke, noto studioso ed editore degli oracoli delfici, il quale, oltre a considerare i quattro versi provenienti da un oracolo sibillino di età classica (redatto nella sua forma definitiva tra la metà del IV sec. e il 146 a.C., data della presa di Corinto da parte dei Romani), rimanda alla perduta Lamia di Euripide ${ }^{32}$.

Diversamente dalla proposta del Casaubon, la ponderata osservazione del Parke può, a mio avviso, trovare conferma, ancora una volta, nella versificazione, quale è attestata dalla tradizione manoscritta. D'altronde, Favorino introduce volentieri citazioni oracolari nella stessa orazione Corinthiaca ( $\S$ 5, p. 303, 23-25 Bar. $^{33}$ ) e nel De exilio (col. 22, 35, p. 403, 9 Bar. $^{34}$ ).

Si raccontava che dalla Sibilla Erofile di Eritrea provenisse una raccolta di profezie scritte in esametri greci, donata anticamente da una vecchia a Tarquinio il Superbo e di cui una copia era conservata nel tempio di Apollo sul Palatino. Tale raccolta andò bruciata nell' 83 a.C., così come la redazione successiva, frutto alquanto eterogeneo per fonti e contenuto, distrutta nel 405

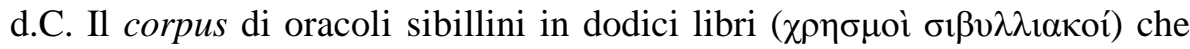

31 Com'è noto, in Pindaro i dattili non hanno una parte molto importante: vedi W.J.W. Koster, «De quibusdam colis dactylicis deque ceteris metris Pindari Pae. IV et IX», Mnemosyne 20, 1967, pp. 396-402.

32 Vedi H.W. Parke, Sibyls and Sibylline Prophecy in Classical Antiquity, ed. by B.C. McGing, London - New York, 1988, pp. 118-119 e 124, n. 32 (ma, per la difficile interpretazione del passo varroniano citato da cui è menzionata la tragedia di Euripide, cf. TGF 506 N. $^{2}$ e il Supplementum (p. 7) di B. Snell). Sembra ora considerare senz'altro un oracolo sibillino la citazione favoriniana anche E. Tagliaferro, «Linguaggi oracoli (sic!) a confronto e la satira di Luciano», in P. Radici Colace - A. Zumbo (a cura di), Atti del Seminario Internazionale di Studi - Letteratura scientifica e tecnica greca e latina (Messina, 29-31 ottobre 1997), Messina, 2000, p. 119 e n. 13, la quale, però, ignora del tutto le problematiche del passo e, conseguentemente, gli studi relativi del Barigazzi.

33 Si tratta di un vaticinium post eventum sulla caduta dei Cipselidi, citato anche in Hdt., V 92. \& 2; A.P. XIV 4.88 e, in maniera incompleta, nello scritto contro gli oracoli di Enomao di Gadara (ap. Euseb., Praep. ev. V 233A): per il valore della testimonianza, cf. H.W. Parke, A History of the Delphic Oracle, Oxford, 1939, pp. 136-137; L. Braccesi, «Le tirannidi e gli sviluppi politici ed economico-sociali», in R. Bianchi Bandinelli (dir.), Storia e civiltà dei Greci, II. Origini e sviluppo della città. L'arcaismo, Milano, 1978, p. 346.

${ }^{34}$ L'oracolo di Apollo, indirizzato ad un certo Lampos di Megara, non meglio identificato, era sconosciuto agli studiosi prima del ritrovamento del papiro contenente la nuova opera favoriniana: cf. Barigazzi, p. 501. 
oggi si legge nella traduzione inglese di R.H. Charles ${ }^{35}$ è quasi completamente un falso, trattandosi per lo più di un'opera di ampliamento ed interpolazione operata all'interno dei circoli giudaici e, in parte, cristiani contro il paganesimo e l'impero romano ${ }^{36}$. La citazione favoriniana, dunque, per dirla col Parke, costituirebbe senz'altro «a very euphuistic composition», oltre che una delle poche testimonianze pagana precedente al corpus sibillino stesso ${ }^{37}$.

Ed infatti, a differenza degli ultimi due versi, esametri regolari, i primi due presentano delle "durezze" metriche o prosodiche che andranno imputate proprio ai bisogni dell'improvvisazione tipica della "poesia" oracola$\mathrm{re}^{38}$, l'unica per la quale gli antichi riconoscano esplicitamente la rozzezza, la banalità e la cattiva qualità della versificazione, ammettendo addirittura casi di esametri acefali, $\mu \varepsilon^{\prime}$ ov $\rho \circ$ e $\lambda \alpha \gamma \alpha \rho o^{39}$.

35 Apocrypha and Pseudepigrapha in the Old Testament, II, Oxford, 1913; vedi, inoltre, Sibylline Oracles (Second Century B.C. - Seventh Century A.D.), a new translation and introduction by J.J. Collins, in The Old Testament Pseudepigrapha, ed. By J.H. Charlesworth, I, London, 1983. Per gli oracoli relativi a Nerone redivivo, si dispone in Italia della versione di F. Sbaffoni, Testi sull'Anticristo. Secoli I-II, Firenze, 1992, pp. 73-101.

${ }_{36}$ Cf. P. Fraser, Ptolemaic Egypt, I, Oxford, 1968, pp. 708-710; G. Luck, Arcana mundi. Magia e occulto nel mondo greco e romano, II. Divinazione, Astrologia, Alchimia, MilanoVerona, 1999, p. 27. Sulla formazione del corpus, vedi A. Rzach, in RE, II, $\mathrm{A}_{2}$, s.v. «Sibyllinische Orakel», coll. 2103-2183; A. Kurfess, Sibyllinische Weissagungen, Berlin, 1951; E. Suárez de la Torre, «Oráculos Sibilinos», in A. Diez Macho (ed.), Apócrifos del antiguo Testamento, III, Madrid, 1982, in part. pp. 241-245; J.J. Collins, «The Development of the Sibilline Tradition», ANRW II, 20, pp. 422-459; E. Schürer, Storia del popolo giudaico al tempo di Gesù Cristo, I-IV, Brescia, 1985-1988.

37 Responsi attribuiti direttamente alla Sibilla sono presenti in Pusania (7, 8, 9; 10, 9, 11 e 12, 3), Plutarco (Dem. 1-2; Thes., 24, 5), Flegonte di Tralles (FGrH 257 F 36). Per una raccolta del materiale pagano, vedi C. Alexandre, Oracula Sibyllina, II, Paris, 1956.

38 Vedi sull'argomento lo studio fondamentale di L.E. Rossi, «Gli oracoli come documento di improvvisazione», in I poemi epici rapsodici non omerici e la tradizione orale, a cura di C. Brillante, M. Cantilena, C.O. Pavese, Padova, 1981, pp. 203-221 (praes. p. 211 e 214); più di recente, L. Maurizio, «Delphic Oracles as Oral Performances: Authenticity and Historical Evidence», ClassAnt 16, 2, 1997, pp. 308-334. Le conclusioni di W.E. McLeod («Oral Bards at Delphi», TAPhA 92, 1961, pp. 317-325, in part. 322), secondo cui gli oracoli resterebbero orali fino al V-IV sec. a.C., sono errate: vedi Rossi, art. cit., pp. 207-208. Per una lista di durezze metriche e prosodiche negli oracoli, cf. H.W. Parke - D.E.W. Wormell, The Delphic Oracle, II. The Oracular Responses, II, Oxford, 1956, pp. XIX-XX; R. Flacelière, Devins et oracles grecs, Paris, 1961, pp. 73-74, 117-118.

39 La testimonianza più preziosa ci viene dal De Pythiae oraculis di Plutarco (in part. 379D e 396C). Si veda anche Luc., Iup. Trag. 6. Quanto alla mancanza di ỏkpíßsı 


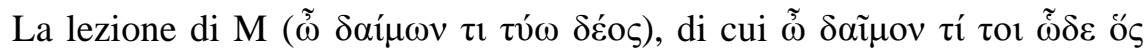
della restante tradizione è una chiara banalizzazione, non ha senso e, per

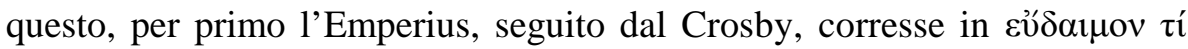

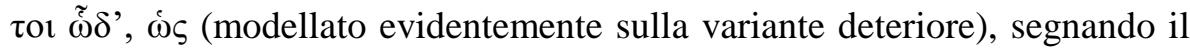
punto interrogativo dopo $\mu$ õ̃vos (v. 4): «Cosa c'è di tanto felice, come... $? \gg^{40}$. L'Arnim, per parte sua, considerando, a ragione, più vicina all'origina-

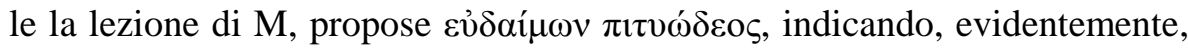

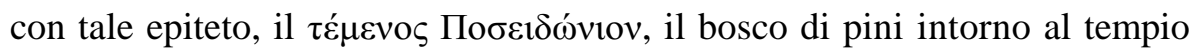
di Posidone, presso il quale erano celebrati gli agoni istmici: cf. Strab., VIII

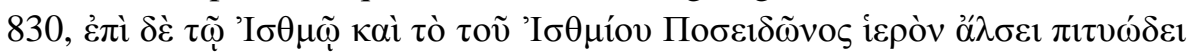

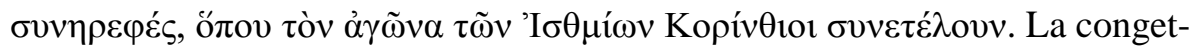
tura è molto seducente, ma, come quella dell'Emperius, non si propone, giustamente come vedremo, di colmare la presunta lacuna metrica del verso. È per questo, credo, che il Barigazzi (seguito dal Davies), stampa tra cruces la lezione di $\mathrm{M}$.

A ben vedere, però, ciò che non va affatto nella ricostruzione dell' Arnim

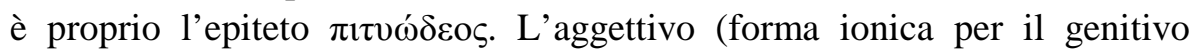
$\pi \iota \tau v \omega \delta \delta o v \varsigma^{41}$ ) resterebbe senza nessun referente: «Fortunato per (qualcosa?) ricoperta di pini, prospero istmo... ». Tutt'al più, dunque, dovrebbe leggersi

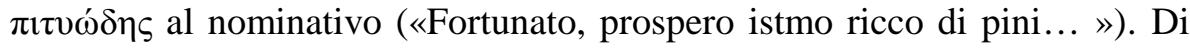
qui la soluzione del Bernabé, il quale, a fronte di varie imprecisioni ${ }^{42}$,

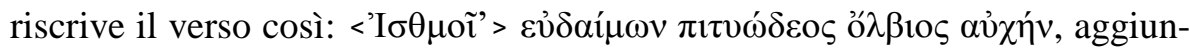
gendo, di suo, una parola affatto testimoniata dalla tradizione manoscritta.

$\mu \varepsilon ́ \tau \rho o v$ per gli oracoli sibillini, essa viene denunciata apertamente nel prologo del corpus sibillino dal suo anonimo autore, che la attribuisce all'incapacità dei $\tau \alpha \chi v \gamma \rho \alpha ́ \varphi o t$, non già alla profetessa (Sib., prol. 83ss.). Tale giudizio deriverebbe, secondo J. Geffcken, Die Oracula Sibyllina, Leipzig, 1902, ad l., da [Just.], coh. Gr. 37, 15. Sull'esametro sibillino, cf. in particolare J.M. Nieto Ibáñez, El Hexámetro de los Oraculos Sibilinos, Madrid, 1992.

${ }^{40}$ La correzione dell'Emperius è alla base anche della traduzione spagnola di G. del Cerro Calderón: «¿Hay lugar tan feliz para ti como...?» (p. 51).

${ }^{41}$ Cf. Bernabé, Poetarum epicorum Graecorum, cit., p. XIII. La forma $\pi \iota v \omega ́ \delta \varepsilon o \varsigma$ per il nominativo non è attestata: vedi $L S J$, p. 1409, s.v. (Suppl., p. 251). Ionismi e forme non

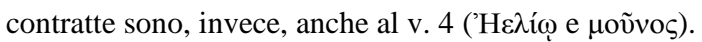

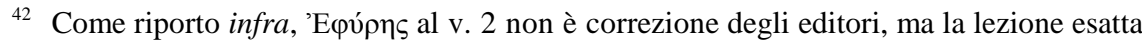

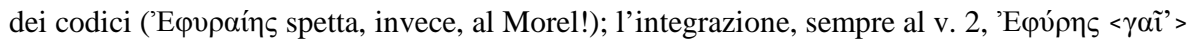
risale al Post e non al Postgate. Per una discussione critica del passo, vedi dello stesso Bernabé il Manual de crítica textual y edición de textos griegos, Madrid, 1992, pp. 96-98. 


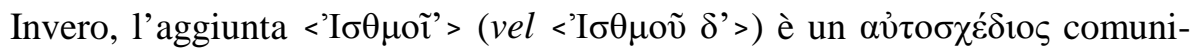
cato oralmente da J. Ebert.

Personalmente, ho già altrove analizzato il verso, proponendo, exempli

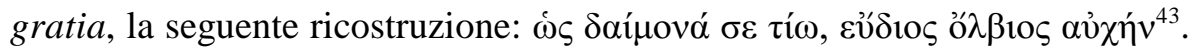
Preferisco, ora, con maggiore convinzione, leggere $\tilde{\tilde{\omega}} \delta \alpha i ́ \mu \omega v \tau \imath \tau i ́ \omega, \delta \tilde{o}$

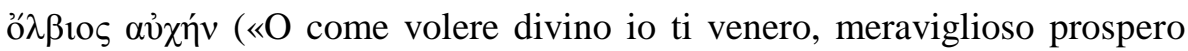
istmo...»), che, oltre ad essere correzione paleograficamente più attendibile, ha, se non altro, il merito di restituire, con degli interventi minimi (omofonia dei suoni $1 / v, 1 / \varepsilon)$, la forma esametrica del verso.

Come avvertivo anche allora, le "brutture" metriche e prosodiche di questo esametro (violazione della cosiddetta legge di Varrone, del divieto, cioè, di bipartizione dopo il terzo metron, e dell' «allungamento metrico» dell'avverbio $\tau$ nel biceps del secondo piede) andranno certo imputate ai bisogni dell'improvvisazione ${ }^{44}$. Esempi simili non mancano nella produzione

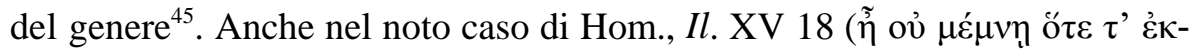

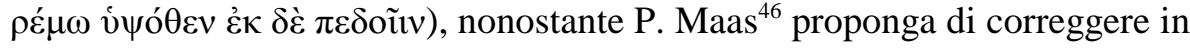
$\tau \varepsilon \kappa \rho \varepsilon ́ \mu \omega$, la violazione della norma della bipartizione è ormai pacificamente

${ }^{43}$ Cf. Amato, Studi su Favorino, op. cit., pp. 54-55. In una recensione al mio libro, apparsa in EMERITA 65, 2, 1997, pp. 358-360, J. Signes Codoñer, che, nonostante le chiare ed 'autorevoli' avvertenze lì riportate, considera la mia proposta «inaceptable, non sólo porque introduce nada menos que siete cambios en el texto transmitido, sino porque además el verso reconstruido no encaja métricamente», congettura $\tilde{\omega} \delta \alpha i ́ \mu \omega v<\alpha ُ \lambda \sigma o v \varsigma>\pi \imath \tau \omega \dot{\delta} \delta \varepsilon$ . L'ipotesi non convince per nulla, sia perché irrispettosa del testo tradito e paleograficamente insostenibile (è introdotta, sulla congettura dell'Arnim, una parola affatto testimoniata, ma ricavata con troppa sicurezza dal locus straboniano sopra riportato, che, a voler ostinatamente difendere, sarebbe da scrivere magari dopo $\pi \imath \tau \omega \omega ́ \delta \varepsilon o \varsigma$ e non prima in modo da spiegarne ragionevolmente la caduta [ $\pi \iota \tau \omega \omega ́ \delta \varepsilon o[~ \alpha ́ \lambda \sigma o v] \varsigma]$; lo studioso, poi, non si accorge che in questo modo introduce anche lui, come me, ben sette cambiamenti!) sia perché la frase non avrebbe alcun senso e resterebbe sospesa: «O divinità del bosco ricoperto di pini (= Posidone), prospero istmo di Efira figlia di Oceano, dove... » (?!). Al contrario, l'irregolarità del verso, così come io lo ricostruisco, è documento della sua versificazione orale e ne fornisce un sigillo di autenticità; né, per questo, viene a soffrirne il senso, che resta chiaro e ben intellegibile.

${ }^{44}$ Non vi è violazione della norma di Wernicke. In effetti, lo spondeo dĩo $\varsigma$ in quarta sede coincide con fine di parola chiusa da sillaba con vocale breve; l'omicron va, tuttavia, considerata lunga dinanzi alla sibilante in quanto seguita da parola che inizia per vocale: cf. Martinelli, op. cit., p. 58.

45 Cf. Rossi, art. cit., p. 211.

46 Metrica greca, traduzione e aggiornamenti di A. Ghiselli, Firenze, $1979^{2}$, § 86. 
accolta. Sarebbero, poi, una prova dell'immediatezza colloquiale del componimento l'uso della seconda persona (chiaramente ricavabile dal contesto ${ }^{47}$ ), caratteristico degli oracoli ${ }^{48}$, e l'accostamento, apparentemente ripetitivo,

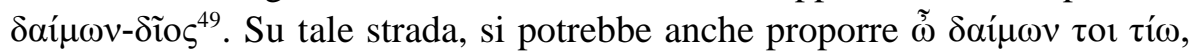

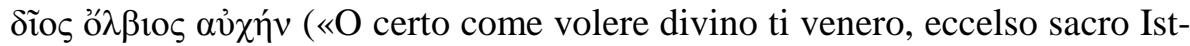
mo...»). La presenza della particella intensiva $\tau$ oı, apparentata, come si sa,

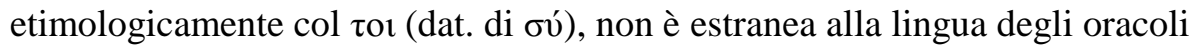
(nell'Index verborum di Parke-Wormell sono segnalati cinque casi) e, sopratutto, caratterizza l' ambito colloquiale e diretto ${ }^{50}$.

Tuttavia, a favore della prima ipotesi vanno gli "omerismi", più o meno formulari, che verrebbero così a caratterizzare il verso $0^{51}$, quale risulta dal mio intervento. In particolare, il verbo tí ('onorare', 'venerare' in particolar modo una divinità) è tipico in Omero e in contesti fortemente indiziari ${ }^{52}$ :

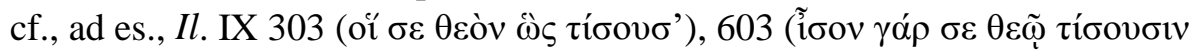

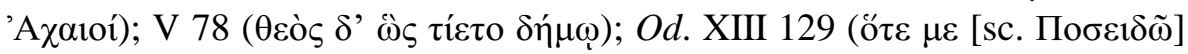

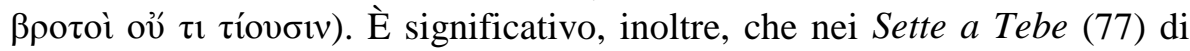

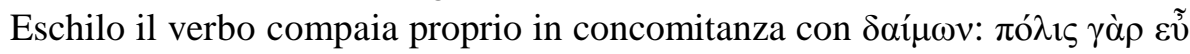
$\pi \rho \alpha ́ \sigma \sigma o v \sigma \alpha \delta \alpha{ }^{\mu} \mu v_{\alpha \varsigma} \tau i ́ \varepsilon 1 . ~ L ' u s o ~ d i ~ \delta \alpha i ́ \mu \omega v$, inoltre, nel senso positivo di 'potenza divina', 'volere divino' legato al singolo avvenimento (Augenblicksgott $\left.{ }^{53}\right)$, com'è la nascita dell'istmo, esalta la sua chiara derivazione da v $\delta \alpha$ ('distribuire', 'dispensare', 'assegnare come parte', 'dare in sorte': vedi $\delta \alpha i ́(\mu \alpha)$, enfatizzando il momento mitico ${ }^{54}$. Per l'impiego, infine, dell'ag-

47 L'ellissi del pronome personale $(\sigma \varepsilon)$, che si può dedurre con facilità dal nominativo di vocazione che precede e che segue, rientra nella figura ảंò кoเvoṽ: cf. Kühner-Gerth, II, pp. 560-561.

${ }^{48}$ Cf. Parke - Wormell, op. cit., p. XXV.

49 Vedi Hes., Th. 991. Sulle ripetizioni negli oracoli, cf., tuttavia, Rossi, art. cit., p. 214.

50 Cf. J.D. Denniston, The Greek Particles, Oxford, 1954², p. 537.

51 Per gli 'omerismi' negli oracoli, vedi lo studio citato del McLeod.

52 La vocale $\mathrm{inel}$ presente è sentita da Omero ora breve ora lunga: cf., e.g., Il. IV 257; IX 238.

53 Cf. H. Usener, Götternamen, Bonn, 1929², p. 280; M.P. Nilsson, Geschichte der Griechischen Religion, I, München, 1941, p. 202, 205; si veda anche M. Untersteiner, Le origini della tragedia e del tragico, rist. Milano, 1984 (Torino, 1955²; Milano, 1942²), pp. 360-361.

${ }^{54} \mathrm{Al}$ termine $\delta \alpha i ́ \mu \omega v$ è ovviamente del tutto estranea la distinzione tra $\delta \alpha i ́ \mu \omega v$ ('essere divino') e $\theta \varepsilon o ́ \varsigma$ ('dio'), che si incontra a partire già da Eschilo e che nella tarda età ellenistica 


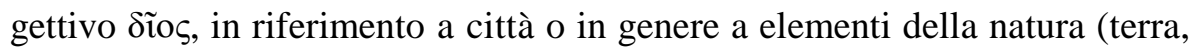
mare, cielo), cf. Hom., Il. I 141; II 615; XIV 347; XVI 365; Od., III 326.

Se veniamo, ora, a considerare il v. 2 , andrà notato come anch'esso presenti una notevole durezza, che può essere metrica (una sillaba in meno) $o$ prosodica (la vocale breve del quinto piede). Eppure, proprio tale 'bruttura' può imputarsi alla redazione originale dell'oracolo, rimasta inalterata nella tradizione e alla quale anche Favorino si attiene: questa fedeltà di trasmissione, come ha chiarito Luigi Enrico Rossi ${ }^{55}$, ha origine «nel considerare il responso oracolare come qualcosa di sacro e di intoccabile» ${ }^{56}$. Per la stessa ragione si giustifica l'inserzione di una sillaba in più nel noto oracolo sul futuro di Sparta trasmesso da Erodoto in VII 220.3 (= 100, 2 Parke-Wormell), difeso a buon diritto da O.J. Todd ${ }^{57}$ e dallo stesso Rossi ${ }^{58}$; esempi simili non dovettero essere, poi, così rari, a sentire Platone (Phdr. 252B). Dif-

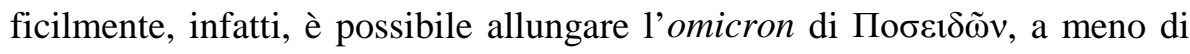
non voler supporre che nella lettura gli antichi abbiano scandito la prima sillaba Пoб-, fino a considerarla chiusa e quindi lunga. Così si avrebbero

finirà con l'indicare quasi esclusivamente le entità demoniche minori (il dèmone) e, quindi, lo spirito maligno (vedi G. Luck, Arcana mundi, op. cit., I. Magia, miracoli, demonologia, Milano-Verona, 1997, pp. 295-297). Nei testi più antichi, come accade anche in Omero, il termine $\delta \alpha i ́ \mu \omega v$ è 'parola divina' usata indifferentemente assieme a $\theta \varepsilon o ́ \varsigma$ per indicare una forza, una potenza divina, superiore agli uomini. Sul concetto di 'parola divina', cf. E. Peterich, Die Theologie der Hellenen, Leipzig, 1938, p. 121; vedi anche Fr. Pfister, Die Religion der Griechen und Römer, Leipzig, 1930, p. 123. Per il valore di $\delta \alpha i ́ \mu \omega v$ in Omero, si vedano in particolare i lavori di S. Tromp De Ruiter, De vocis quae est $\triangle \mathrm{AIM} \Omega \mathrm{N}$ apud Homerum significatione atque usu, Amstelodami, 1918; M.P. Nilsson, «Götter und Psychologie bei Homer», Arch. Religionswiss. 22, 1923-1924, pp. 377-380 (ora in Opuscula selecta, I, Lund, 1951, pp. 374-377); Wilamowitz-Möllendorff, op. cit., I, pp. 362-363; M. Untersteiner, «Il concetto di $\delta \alpha i ́ \mu \omega v$ in Omero», $A \& R$ s. III, 7, 1939, pp. 93-134; U. Bianchi, $\triangle \mathrm{IO \Sigma}$ AIsA. Destino, uomini e divinità nell'epos, nelle Teogonie e nel culto die Greci, Roma, 1953, pp. 115-132.

55 Art. cit., p. 206-207.

56 Dalla testimonianza solidale di Erodoto (VI 57.2) e di Senofonte (Lac. 15.5) sappiamo, ad esempio, che a Sparta esistevano dei veri e propri funzionari cui spettava il compito di conservare i testi dei responsi (cf. Parke - Wormell, op. cit., pp. XIV-XV). Inoltre,

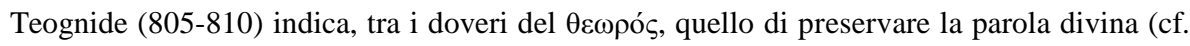
Parke - Wormell, op. cit., p. XXXIII).

57 «An inelegant Greek Verse», CQ 33, 1939, pp. 163-165.

58 Art. cit., p. 208. 
spondaiche quarta e quinta sede, errore "ammesso" nella prosodia oracola$\mathrm{re}^{59}$. Più equo attribuire il difetto di una sillaba alla versificazione frettolosa ed alla tecnica improvvisata della letteratura del genere.

Solo il von Arnim, che io sappia, seguito dal de Budé, ha stampato il verso così come è tràdito dai codici, senza, tuttavia, fornire ulteriori chiarimenti. Al contrario, a partire dal Morel, l'intervento minimo 'E $\varphi$ voín

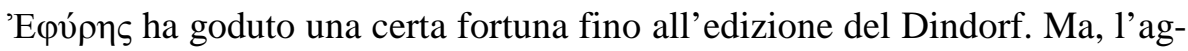
gettivo è nome della terra, non certo della ninfa; la proposta è, pertanto, errata $^{60}$. Questo, in buona sostanza, ha visto il Crosby, il quale ha inserito un' integrazione di L.A. Post: $\gamma \alpha \tilde{\imath}$ ’ $\varepsilon ́ v \theta \alpha$ (“terra dove..."). La correzione, discutibile dal punto di vista paleografico, è molto meno convincente rispetto a quella dello stesso Barigazzi $(\dot{\varepsilon} v<\tau \alpha \tilde{v}>\theta \alpha)$, che, anche se seducente, richiede,

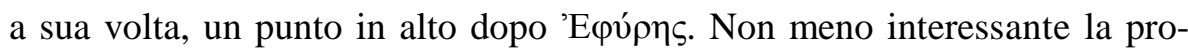
posta di J. Ebert, esemplificata su Hom., Il., IX 194 e accolta dal Bernabé, di

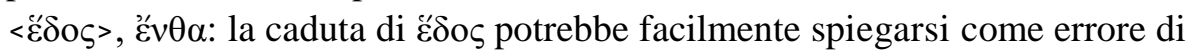

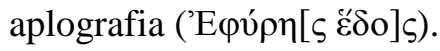

Come che sia, i dati metrici finora rilevati per entrambe le citazioni favoriniane escludono una possibile derivazione dai Korinthiaká di Eumelo, facendo piuttosto propendere, con una certa verosimiglianza, per un frammento lirico di autore incerto ${ }^{61}$ e per un oracolo sibillino del periodo classico.

59 Vedi 31, 1; 318, 2; 473, 20 Parke-Wormell.

60 Cf. Barigazzi, p. 323. Per lo stesso motivo, non va accolta la correzione del West (ap.

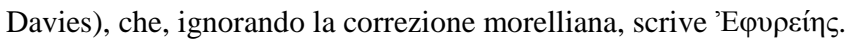

61 Per una possibile attribuzione, vedi infra. Favorino nelle sue opere mostra una certa predilezione per le citazioni e le parafrasi di luoghi poetici. Era questa del resto un'abitudine della neosofistica, consigliata dal retore Ermogene (Id., p. 362, p. 20ss. Rabe), sui cui si veda almeno A. Boulanger, Aelius Aristide et la sophistique dans la province d'Asie au II siècle de nôtre ère, Paris, 1923 (rist. 1968), pp. 440-445; V.A. Sirago, «La seconda sofistica come espressione culturale della classe dirigente nel II sec.», ANRW II, 33.1, 1989, in part. pp. 7274; E. Bowie, «Greek Sophists and Greek Poetry in the Second Sopistic», ibid., in part. pp. 210-214 (e relativa bibl.); L. Spina, «Ermogene e la citazione poetica», in AA.VV., 'Come dice il poeta'. Percorsi greci e latini di parole antiche, Napoli 1992; L. Pernot, La rhétorique de l'éloge dans le monde gréco-romain, II. Les valeurs, Paris, 1993, pp. 726-738. Se si esclude Plutarco, rispetto ad altri sofisti (Luciano, Elio Aristide, Massimo Tirio, Dione Crisostomo, Polemone, Erode Attico, Lesbonatte), meno interessati nelle loro opere ai poeti 
A questi elementi formali (di per sé già necessari e sufficienti) si possono aggiungere alcune osservazioni di carattere storico-letterario, che portano a ritenere fondata l'opinione che Favorino, nel luogo che stiamo studiando, non abbia introdotto citazioni di Eumelo ${ }^{62}$.

Un aiuto importante ci viene proprio dal testo di Pausania, invocato all'inizio del contributo, che, contrariamente a quanto ritenuto dal Barigazzi, non permette, ad una lettura attenta, di inferire che Eumelo sia l'autore delle due citazioni in esame.

arcaici (eccezion fatta, s'intende, per Omero, continuamente in mano agli scrittori di età imperiale: vedi J.F. Kindstrand, Homer in der Zweiten Sophistik. Studien zu der Homerlektüre und dem Homerbild bei Dio von Prusa, Maximos von Tyros und Ailios Aristides, Uppsala, 1973; O. Bouquiaux-Simon, Les lectures homériques de Lucien, Bruxelles, 1968), Favorino appare, in rapporto a quanto è sopravvissuto, molto più aperto agli autori del periodo ionico, come dimostrano le continue citazioni di Alceo, Saffo, Pindaro, Anacreonte (è da lamentare l'assenza di un Index locorum nella pur corposa edizione del Barigazzi): si vedano, di recente, sul problema e per nuove ricognizioni, Tepedino Guerra, Un nuovo frammento pindarico dell'Inno ad Ammone?, cit.; Ead., «Un nuovo frammento di Alceo nel de exilio di Favorino?», di prossima pubblicazione negli Studi in onore di Italo Gallo, a cura di L. Torraca e P. Volpe Cacciatore, Università degli Studi di Salerno; Amato, «Cristoph M. Wieland lettore di Luciano», cit., pp. 113-117. Reminiscenze varie sono state segnalate in Cor., 2, p. 303, 5-6 Bar. (cf. Crosby, op. cit., p. 7, n. 1; Barigazzi, p. 315; Amato, Studi su Favorino, cit., pp. 47-48; del Cerro Calderón, op. cit., p. 46, n. 5: Pi., fr. 124, 1 e 140b, 8 Sn.-M.); 20, p. 307, 1-2 (cf. Casaubonus, op. cit., p. 74; Reiske, op. cit., II, p. 460, n. 52; Emperius, Opuscula philologica et historica, op. cit., p. 41; Barigazzi, p. 328; Amato, Studi su Favorino, op. cit., p. 59: tragedia [?]); 47, p. 313, 12-13 (cf. J.M. Edmonds, Lyra Graeca, I, Oxford, 1922, p. 236; Crosby, op. cit., p. 47, n. 4; del Cerro Calderón, op. cit., p. 71, n. 100: Saffo; Barigazzi, p. 346: tragedia); de fort., 14, p. 257, 19-20 Bar. (cf. Amato, «Nuova eco saffica in Favorino?» in appendice a «Cristoph M. Wieland lettore di Luciano», cit., p. 124-125: Sapph., fr. 132 V.).

62 Di passaggio, vorrei sottolineare che, almeno per quanto riguarda il pentametro lirico su Briareo, l'ipotesi del Barigazzi, che privilegia esclusivamente i Korinthiaká di quel poeta, risulta metodologicamente male impostata, dal momento che del mitico centimano Eumelo avrebbe potuto fare menzione anche nella sua perduta Titanomachia, in cui Briareo era certamente di casa. Che Eumelo, infatti, fosse autore di una Titanomachia è detto chiaramente da Igino (fab. $183=$ Titanom. F 7 Bernabé; $4^{\mathrm{B}}$ Davies) e da Ateneo (I 22C $=$ F 6 Bernabé; 5 Davies; VII 277D = F 4 Bernabé; 8 Davies). In particolare, la testimonianza di $\Sigma$ Ap. Rhod. I 1165 (F 3 Bernabé; 3 Davies) lascia intendere che Eumelo nella sua opera introducesse, assieme agli altri centimani, Briareo, il quale compariva anche in altre opere analoghe (vedi ¿Pind., Nem. 3.40 [III 48, 10 Drachm.] = F 16 Bernabé; adesp. 6 Davies). 
Pausania introduce, nel corso della sua opera, sei volte Eumelo: tre nel secondo libro (quello relativo alla Corinzia), due nel quarto (dedicato alla Messenia), una nel quinto (Olimpia).

Negli ultimi tre casi, la testimonianza del periegeta è di fondamentale importanza per recuperare alcuni versi del già citato prosodion in onore dell'Apollo di Delo, che, secondo C.O. Pavese, rappresenterebbero il più antico frammento di lirica corale greca. Ciò che, in particolare, risulta funzionale al nostro discorso è quanto Pausania scrive in IV 4.1: «Sotto Finta figlio di Sibota, per la prima volta i Messenii inviano all'Apollo di Delo un sacrificio e un coro di uomini: fu Eumelo a istruire per loro il canto pro-

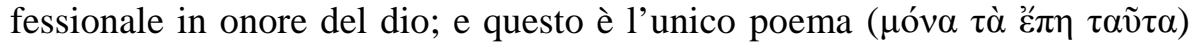
che si ritiene essere davvero opera di Eumelo» (trad. D. Musti).

In effetti, nell'esordio del secondo libro (II 1.1 = T 1 Bernabé; 1 Davies), Pausania dice di aver attinto materiale, per la descrizione della regione, da

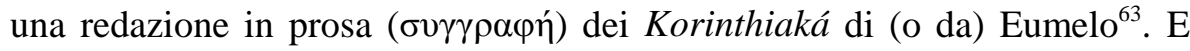
che si tratti di tradizione scritta è confermato da Pausania stesso in II $2.2 \mathrm{e}$ 4.1, dove sono usate le espressioni $\dot{\varepsilon} \pi \imath \lambda \varepsilon \xi \alpha \dot{\alpha} \mu \varepsilon v o \varsigma$ e $\dot{\varepsilon} \pi \varepsilon \lambda \varepsilon \xi \alpha \dot{\alpha} \mu \eta \nu$.

Ora, come è stato giustamente osservato, dal momento che lo scrittore mostra di avere una conoscenza abbastanza precisa del prosodion a Delo, di cui menziona i versi in IV 4.1 e 33.2, è facile concludere che i Korinthiaká siano noti a Pausania solo nella versione in prosa allora circolante; anzi, che lo stesso prosodio fosse citato in quella versione prosastica ${ }^{64}$. Sembra curioso, infatti, che il periegeta non citi neppure una volta nel libro della Corinzia (dove la testimonianza di Eumelo per la ricostruzione della storia di Corinto antica risulta di fondamentale importanza almeno nei primi tre capitoli) $\mathrm{i}$ suoi Korinthiaká, neppure un solo verso.

È nota la cura e l'attenzione di Pausania nella scelta e nell'impiego delle fonti, non solo, naturalmente, per abbellire e ravvivare il contenuto della sua

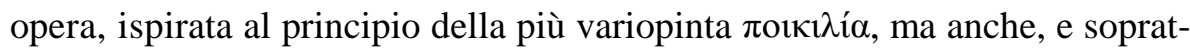
tutto, per avvalorare le tesi di volta in volta riportate. Ha scritto il Musti: «Poeti come Omero e, probabilmente, Pindaro valgono per lui come fonti di

63 Sul valore di tale redazione, che doveva rispettare fedelmente la narrazione originale in poesia, vedi G. Marckscheffel, Hesiodi, Eumeli, Cinaethonis, Asii et carminis Naupacti fragmenta, Lipsiae, 1840, pp. 223-225.

${ }^{64}$ Cf. Pavese, art. cit., p. 54. 
particolare attendibilità, per la loro antichità e autorevolezza: la loro testimonianza è talora citata come decisiva, quando altre contrastano» ${ }^{65}$. Sotto questo aspetto, lo scrittore risulta «cultore del raro e del prezioso, di ciò che sfugge ai più». Una rapida scorsa agli indici della Descriptio ci mostra la faccia quanto mai ricca di tutta una letteratura minore: Pausania cita le Eoie, i Canti Ciprii, la Miniade, i Nostoi, la Piccola Iliade, i Canti Naupattii, la Tesprotide, l'Edipodia, la Tebaide, i poeti Lescheo, Cintone, Aristea, Asio, Paniassi, Apollonio, Euforione, ecc.; ed è in grado di distinguere tra Eoie e Grandi Eoie, di discutere dell'autenticità di alcuni versi di Orfeo, Museo, Filammone di Argo. Tali informazioni sono frutto, certo, delle sue letture, ma anche delle esperienze dirette delle regioni descritte. E così, anche per il II libro della Descriptio, accanto alle citazioni di tradizione scritta (tra cui,

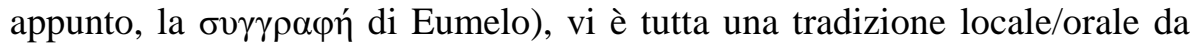
non sottovalutare. Anzi, si può ben dire che la descrizione di Corinto antica comincia proprio nel segno del contrasto di opinioni tra gli abitanti della città (con chiaro riferimento a tradizioni orali) e la storia scritta da Eumelo, che l'autore sembra preferire ${ }^{66}:$ «La terra corinzia è parte della terra argiva ed ha nome da Corinto. Nessuno, ch'io sappia, ha mai sostenuto seriamente che Corinto fosse figlio di Zeus, all'infuori della maggior parte dei Corinzi

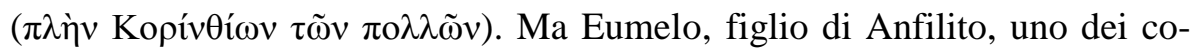
siddetti Bacchiadi, che si dice abbia composto anche il poema epico, afferma nella storia corinzia (se pure è sua questa storia), che in questa regione abitò dapprima Efira, figlia di Oceano, e che poi Maratone (...) si trasferì sulla costa dell'Attica; morto il padre, venne nel Peloponneso, divise il regno tra i figli e se ne ritornò in Attica: la terra Asopia ricevette il nome di Sicione, la terra Efirea quello di Corinto» (trad. Musti).

${ }^{65}$ Così D. Musti, Pausania. Guida della Grecia, Libro I. L’Attica, Milano-Verona, $1987^{2}$, p. XXIV. La rivalutazione della Periegesi di Pausania, su cui è pesato per qualche tempo il giudizio alquanto negativo del Wilamowitz (Götting. Gelehrte Anzeiger, 1906, p. 638 = Kleine Schriften, Berlin, V, p. 373), che riteneva le informazioni presenti in essa secondarie e quasi mai suffragabili, è concordemente ribadita dalla critica recente: vedi, tra le monografie più vicine, J. Heer, La personalité de Pausanias, Paris, 1979; Ch. Habicht, Pausanias' Guide to Ancient Greece, Berkeley - Los Angeles - New York, 1985 (esiste anche un'edizione tedesca apparsa in contemporanea con il titolo Pausanias und seine Beschreibung Griechenlands, München, 1985). Per un punto sulla questione, cf. G. Maddoli, in A.M. Biraschi - G.M., «La geografia: Strabone e Pausania», in AA.VV., Lo spazio letterario della Grecia antica, I, 3, Roma, 1994, pp. 197-200.

${ }^{66}$ Cf. Musti, op. cit., Libro II. La Corinzia e l'Argolide, Milano-Verona, 1986, p. 205. 
In effetti, anche quando Pausania usa espressioni quali $\lambda \varepsilon ́ \gamma o v \sigma ı, ~ \varphi \alpha \sigma i ́$ con specifico soggetto Kopív $\theta$ tor, sembra equo ritenere che in questo modo egli focalizzi l'attenzione del lettore su tradizioni orali opposte a tradizioni sicuramente scritte ${ }^{67}$. Per i casi di citazioni di versioni accreditate dai Corinzi, non già dal poeta epico, la cui opera sembra offrire piuttosto una sistemazione e una manipolazione della storia mitica di Corinto, ricordo i loci di $1.6 \mathrm{e}$ 4.6, alquanto indicativi per il nostro discorso. Da questi passi si ricava che le informazioni, cui accenna anche Favorino nella Corinthiaca, relative alla gara tra Posidone ed Elio, giudice Briareo, per il possesso del territorio, risalgono ad una tradizione orale e non ad Eumelo come avviene per i miti discussi in 1.1, 2.2, 3.10-11.

Ebbene, alla luce di quanto finora detto, la testimonianza di Pausania, contemporaneo o di poco più giovane di Favorino ${ }^{68}$, deve farci ulteriormente riflettere sulla reale possibilità che i versi di questo derivino dai Korinthiaká di Eumelo: se all'epoca di Favorino fosse circolata ancora una copia in versi dell'opera epica del poeta corinzio, probabilmente questa non sarebbe rimasta sconosciuta a Pausania e, dunque, inutilizzata. Piuttosto, forse, è da pensare che nell'età di Adriano ed Antonino il Pio, l'unica versione disponibile, che anche Favorino ha potuto tranquillamente tener presente per il suo discorso, sia proprio quella prosastica menzionata esplicitamente da Pausania. Ma questo non autorizza in nessun modo a concludere che le due citazioni poetiche favoriniane siano da attribuire ad Eumelo. Possiamo solo dire che l'autore (dell'epitome) dei Korinthiaká potrebbe rappresentare, probabilmente assieme ad altri storici e scrittori di cose locali ${ }^{69}$, una delle fonti

67 Cf. Musti, ibid., p. 221.

68 Pausania vive sotto il regno di Adriano, Antonino il Pio e Marco Aurelio, per un arco di vita, abbastanza lungo, che cade tra il 100/110 d.C. e il 180 ca. Per la datazione, in particolare, del libro II, la cui cronologia oscilla tra il 150 ca. e il 170 ca., vedi Musti, op. cit. (nota 63), pp. XXXI-XXXII. Favorino nasce, invece, intorno all'80/85 d.C. e muore verso il 165/170 (cf. Amato, «Cristoph M. Wieland lettore di Luciano», art. cit., p 105-106, n. 3) e la sua Corinthiaca va ascritta al 130.

69 Oltre a storie locali, quali quelle di un certo Teseo (FGrHist 453) e di un Dioxippo corinzio (FGrHist 454), esistevano cataloghi di vincitori e scritti dedicati esclusivamente ai giochi dell'Istmo: si pensi al $\pi \varepsilon \rho \grave{~ ' I \sigma \theta \mu i ́ \omega v ~ d i ~ E u f o r i o n e ~ d i ~ C a l c i d e ~(v e d i ~ A t h e n ., ~ I V ~ 182 E ~ e ~}$ 183F; XIV 635F e Plut., quaest. conv. V 3.3, il quale menziona anche un Procles, da

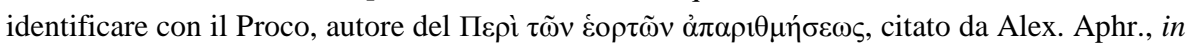
$S E$, p. 9 Wallies). Un tale Musaeus, autore di un'opera analoga, è ricordato, inoltre, in $\Sigma$ Ap. 
utilizzate dal retore per enfatizzare, dinanzi al suo uditorio, le origini divine della città di Corinto e delle gare istmiche colà celebrate.

Non voglio, ora, concludere senza portare una timida ipotesi (che come tale deve essere riguardata) sull' autore del pentametro lirico a Briareo.

In casi come questi, per citare le parole illustri del Nauck, lubrica est via quam ingrediuntur qui poëtarum reliquiis operam navarint...; voler tentare, dunque, di identificare il contesto di appartenenza è fatica di Sisifo.

Tuttavia, possono essere in qualche modo da guida la lingua e il metro del frammento, che, come ho ricordato più sopra, sembra essere tipico del nomos citarodico. A sua volta, la forma attica $\chi \varepsilon i \bar{\rho} \varepsilon \varsigma$ dovrebbe assicurarci che la citazione non proviene né da Stesicoro ${ }^{70}$ né da Ibico ${ }^{71}$, i quali, in base ai frammenti superstiti, adottano il dialetto dorico impastato con una ricca dose di elementi ionici, in omaggio alla dizione epica tradizionale ${ }^{72}$. Anzi, andrà senz'altro segnalato che Stesicoro utilizza in S 11.1 l'iperdorico $\chi \eta \rho \sigma i v^{73}$.

Perché, allora, non pensare, tra gli autori che hanno fatto uso di questo tipo di pentametro, proprio ad Eschilo ${ }^{74}$ autore di una tetralogia sul mito degli Eolidi, che portava in scena, probabilmente come dramma satiresco, i

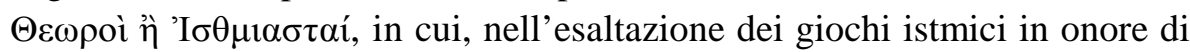
Melicerte, veniva placato lo spirito dei morti figli di Atamante ${ }^{75}$ ?

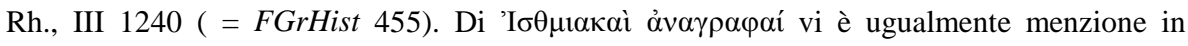
¿Pind., Isth. 1.11.

70 Verrebbe fatto di pensare per evidenti ragioni agli "A $\theta \lambda \alpha$ غ̇ं

71 Ibico, come Stesicoro, mostra un grande interesse per le gare sportive, tant'è che Ateneo non sapeva se attribuire a lui, piuttosto che all'altro, i già citati Giochi per Pelia. Ricordo che Ibico menziona il centimano Briareo in fr. 45.

${ }^{72}$ Per la lingua di Stesicoro e Ibico, vedi in particolare M. Nöthiger, Die Sprache des Stesichorus und des Ibycus, Zürich, 1971. Cf., inoltre, F. De Martino, Stesicoro con un lessico dialettale, Bari, 1984, pp. 13-21.

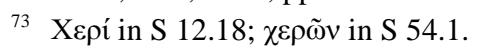

${ }^{74}$ Favorino, in linea con gli autori della Seconda Sofistica, privilegia tra i tre tragici le citazioni da Euripide; un verso delle Coefore di Eschilo è, però, menzionato in de ex., col. 10, 42.

75 Dell'Atamante restano soli due frammenti certi (ffr. 1-2 Radt = 11-12 Mette). L'ipotesi di F. G. Welcker (op. cit. infra) secondo cui all'Atamante fossero legati gli Spettatori è ripresa da H.J. Mette (Die Fragmente der Tragödien des Aischylos, Berlin, 1959, p. 5; Id., Der 
La trama di questo dramma ${ }^{76}$, noto finora solo attraverso un felice ritrovamento papiraceo (fr. 78 Radt $=17$ Mette) e una citazione di Fozio (fr. 79 Radt $=20$ Mette), è stata parzialmente restituita ${ }^{77}$. A noi non interessa naturalmente affrontare in maniera capillare il problema della ricostruzione dell' opera eschilea né quello della tetralogia a cui apparteneva (e, dunque, se si trattava di una tragedia o di un dramma satiresco): è noto che la questione non è semplice e presenta notevoli difficoltà. Basterà ripercorrere, almeno

verlorene Aischylos, Berlin, 1963, pp. 164-170), che considera, come pare da tempo definitivo, l'opera un dramma satiresco (vedi anche V. Steffen, Satyrographorum Graecorum reliquiae, Posnaniae, 1935, ffr. 22-25; B. Snell, Szenen aus griechischen Dramen, Berlin, 1971, p. 37). Per la formazione della tetralogia, di cui questo dramma faceva forse parte (Atamante Epigoni - Teori - Sisifo fuggitivo), si veda lo studio approfondito di M. Untersteiner, «La tetralogia eschilea degli Eolidi», Dioniso 13, 1950, pp. 3-21; 14, 1951, pp. 3-29 (ora in Da Omero ad Aristotele. Scritti minori - seconda serie, Brescia, 1976, p. 177-227), sui cui limiti vedi Q. Cataudella, «La ricostruzione delle tragedie greche perdute», $C \& S$ 1967, pp. 37-38 (= Saggi sulla tragedia greca, Messina-Firenze, 1969, pp. 421-422).

${ }^{76}$ L'ipotesi corrente, alla quale anche io mi conformo, è che si tratti di un dramma satiresco (vedi la bibliografia cit. nelle nn. 73 e 76); tuttavia, non sono mancate le voci contrarie: oltre il fondamentale lavoro di Untersteiner, cf. F.G. Welcker, Die aeschylische Trilogie Prometheus ... nebst Winken über die Trilogie des Aeschylus überhaupt, Darmstadt, 1824, p. 339; E.A.I. Ahrens, Aeschyli Tragoediae septem et perditarum fragmenta..., Parisiis, 1864, p. 232a; A. Tovar, rec. a E. Lobel, The Oxyrhynchus papyri, Part XVIII, London, 1941, EMERITA 11, 1943, p. 438 e 440. In particolare, il Tovar suppone che la tragedia riguardasse la fine dei Molionidi (figli ad un tempo di Attore e di Posidone) per mano di Eracle, mentre si recavano come $\theta \varepsilon \omega \rho$ oí all'Istmo. Ma l'ipotesi è vox clamans in deserto.

$77 \mathrm{Si}$ vedano, a vario titolo, gli importanti contributi di R. Cantarella, I nuovi frammenti eschilei di Ossirinco, Napoli, 1948, pp. 71-99; Untersteiner, «La tetralogia eschilea degli Eolidi», cit., in part. pp. 199-219; Id., Le origini della tragedia e del tragico, op. cit., p. 325330; A. Setti, «Eschilo satirico II», ASNP 21, 1952, pp. 205-244 (ora in Eschilo satirico e altri saggi, Roma, 1981, pp. 82-123); B. Snell, «Aischylos' Isthmiastai», Hermes 84, 1956, pp. 1-11; M. Di Marco, «Studi sul dramma satiresco di Eschilo», Helikon 1969-1970, pp. 373-422; Id., «Sul finale dei 'Theoroi' di Eschilo: fr. 78c, 37ss. R.», Eikasmos 3, 1992, pp. 93-104; I. Gallo, «Ricerche su Eschilo satiresco», in AA.VV., Studi salernitani in memoria di Raffaele Cantarella, Salerno, 1981, pp. 97-155 (ora in Ricerche sul teatro greco, Napoli, 1992, pp. 43-94); D.F. Sutton, «Aechylus 'Theoroi or Isthmiastai': A Reconsideration», GRBS 22, 1981, pp. 335-338, A. Merlo, «Notas a los Theoroi de Esquilo», in A. López Férez (ed.), De Homero a Libanio. Estudios actuales sobre textos griegos, II, Madrid, 1995, pp. 5771; P. O'Sullivan, «Satyr and image in Aeschylus' Theoroi», CQ 50, 2, 2000, pp. 353-366. Cf., inoltre, M. Pohlenz, La tragedia greca, trad. it. Brescia, $1978^{2}$, p. 160; U. Albini, Nel nome di Dioniso. Vita teatrale nell'Atene classica, Milano, 1991, pp. 207-208. 
nell'essenziale, il contenuto dei frammenti superstiti, per concentrare l'attenzione sulla possibile attribuzione a tale dramma del pentametro lirico favoriniano.

Dai resti che abbiamo si lascia intravedere per certo la presenza di un coro di spettatori (theoroi), con molta probabilità i Satiri oppure una delegazione inviata a Corinto da un'altra città (Atene?), che sta per celebrare $\mathrm{i}$ giochi istmici in onore di Positone, al quale il coro si rivolge. I theoroi portano in omaggio al dio, come oggetti votivi, maschere così ben fatte, fatte nello stile di Dedalo, da sembrare reali. Interviene, a questo punto, un nuovo personaggio (Dioniso?), che apostrofa il coro per aver abbandonato la danza a favore dei nuovi giochi, finendo con l'attribuire così al pino maggior onore che non all'edera. Alla fine, dopo uno scambio di battute pungenti, i personaggi del coro si arrendono alle ragioni del deuteragonista e tutti insieme si avviano ad inaugurare $i$ giochi istmici.

Nulla di più facile che durante il dramma vi fosse menzione della fondazione dei giochi ad opera di Posidone a seguito della contesa con Elio per il possesso dell'Istmo, giudice Briareo. Mi inducono a questa conclusione, oltre la trama generale del dramma (almeno per quello che si lascia intravedere con maggiore chiarezza), due elementi fondamentali. Anzitutto, la descrizione da parte di Eschilo della misteriosa statua votiva, offerta a Posidone dal personaggio indicato generalmente con la lettera $\mathrm{A}$, che, per la sua stra-

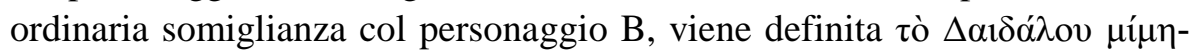
$\mu \alpha$, alla quale $\varphi \omega v \tilde{\eta} \varsigma \delta \varepsilon \tilde{~} \mu o ́ v o v$.

Ora, senza stare a elencare le fonti storico-artistico-letterarie che sono alla base di questa definizione dell'arte di Dedalo, sarà un caso che nel passo favoriniano in cui è inserito il pentametro lirico vi sia menzione proprio di Dedalo e della sua straordinaria capacità di riprodurre statue moventi, del tutto simili al vivo personaggio in esse rappresentato? L'intero locus della fondazione dei giochi istmici, collegati al mito della fondazione di Corinto,

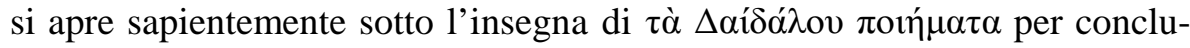

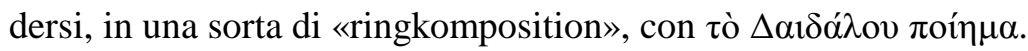

Ancora, è degno di attenzione nel testo di Favorino la citazione dell'epigramma attribuito ad Orfeo, in cui si parla di una corona di pino assegnata quale premio ai vincitori delle Istmie ('I $\sigma \theta \mu 1 \alpha \kappa \alpha \lambda \lambda$ 1кó $\tau v \sigma v)$. Che si tratti di una tradizione antica e primordiale, legata alle vicende della protostoria di Corinto, presente e quasi operante nella mente di Fa- 
vorino, raccoglitore e cultore di notizie rare, non solo in qualità di erudito, ma anche come sapiente retore, troverebbe, guarda caso, risalto proprio dal confronto con un altro frammento degli ' $\mathrm{I} \sigma \theta \mu \iota \alpha \sigma \tau \alpha$ di Eschilo, in cui Dioniso (ovvero il personaggio $\Gamma$ ) rimprovera al personaggio $B$ di trascurare la danza per seguire un atletismo diverso, ginnico, che si pratica ai giochi dell'

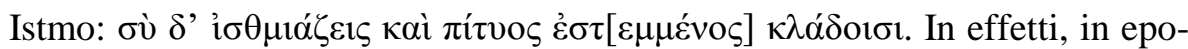
ca recente, il premio della vittoria istmica consisteva, ad imitazione delle Nemee, in una corona di apio fresco, in sostituzione dell'altra di pino molto più antica ${ }^{78}$. Ad assicurarcelo è anche Callimaco, lettore infaticabile e fonte preziosa di tradizioni ipomnematiche e scoliografiche, che negli Aitia (fr. 59 Pf. = Suppl. Hell. 265), echeggiando, come ha messo in luce R. Pfeiffer ${ }^{79}$, proprio il frammento eschileo degli 'I $\sigma \theta \mu 1 \alpha \sigma \tau \alpha$ í, parla della corona di pino

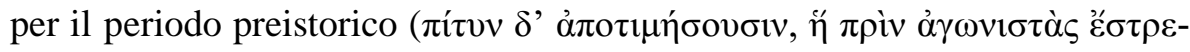

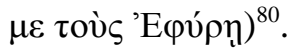

Ecco così che, se la mia ipotesi dovesse cogliere nel vero, avremmo rinsaldati fra loro gli anelli di una catena poetico-erudita trasmessa da Eschilo e Callimaco e continuata da Favorino, il cui testo ci consentirebbe, ancora una volta, di recuperare un nuovo frammento alla lirica greca.

In ogni caso, sia che il pentametro lirico a Briareo debba farsi risalire ad Eschilo o meno, non v'è dubbio che entrambe le citazioni favoriniane non appartengono, come voleva il Barigazzi e come finora si è ritenuto certo, ai Korinthiaká di Eumelo.

78 Cf., e.g., $\Sigma$ Pi., Isth., vol. III, p. 193 Dr.; Luc., Anac. 9 e soprattutto Plut., quaest. conv. $\mathrm{V} 3$, dove, alla luce della una citazione callimachea che di seguito riporto ed una di Euforione (fr. 84 Pow.) si discute del perché alle Istmie col tempo sia invalsa la norma di coronare i vincitori con l'apio e non più col pino. Sull'argomento vedi J.H. Krause, Die Pythien, Nemeen und Isthmien, Leipzig, 1841 (fotorist. Hildesheim, 1975), pp. 49-40; O. Broneer, «The Isthmian Victory Crown», Amer. Journ. of Arch. 66, 1962, pp. 259-261.

79 Kallimachosstudien, München, 1922, p. 63. Cf. anche G. Capovilla, Callimaco, II, Roma, 1967, pp. 162-163.

${ }^{80}$ Cf., inoltre, Call., fr. 384, 3 Pf. (Epinicio per Sosibio), dove, secondo la ricostruzione

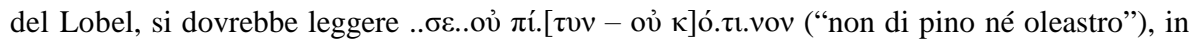
riferimento ai tipi di corona precedenti quella di apio con cui venivano incoronati i vincitori alle gare Istmiche. 